\title{
Modeling and Measuring the Shielding Effectiveness of Carbon Fiber Composites
}

\author{
Luis D. Angulo, David Escot Bocanegra, Patricia Gómez de Francisco, Borja Plaza Gallardo, David Poyatos \\ Martinez, Miguel R. Cabello, Salvador G. Garcia, Senior Member, IEEE
}

\begin{abstract}
We provide a model able to predict the shielding effectiveness (SE) of carbon fiber composite (CFC) panels made of stacked layers of conducting fibers. This model permits us to obtain simple formulas in which the only parameters needed are the sheet square resistance and the effective panel thickness. These tools let us to predict a minimum SE, which always increases with the frequency and therefore constituting the worst case, from an electromagnetic shielding perspective. Consequently, the measurement of minimum SE requirements can be simply measured with a micro-ohmmeter using an specific experimental setup which is also described here. Additionally, this method allows to measure very high SE falling far beyond the dynamic range of the values measurable with the most commonly used standard, the ASTM D4935. After describing the modeling technique and the different test setups used, a cross-validation between theoretical and experimental results is made for four different samples of CFC; two designed to test the modeling assumptions and two which are representative of the ones nowadays used in a real aircraft.
\end{abstract}

Index Terms-carbon fiber composites, electromagnetic compatibility, shielding effectiveness, thin-layer modeling

\section{INTRODUCTION}

$\mathbf{I}$ N RECENT TIMES carbon fiber composite (CFC) panels have earned a larger share as design materials in many industries, which greatly appreciate their mechanical properties [1]. However, from an electromagnetic compatibility (EMC) perspective, CFCs have inferior capabilities when compared to the metals that they usually replace [2]. For this reason, it is convenient to adequately model their electromagnetic shielding effectiveness (SE) in order to ensure an adequate protection for the different systems in which they are used. Even when their mechanical characteristics are well-known, their electromagnetic behavior presents many subtleties which arise from their filamentary and stock-piled geometry. On top of this, they pose an intrinsically multi-scalar problem when they must be incorporated into simulations which involve an entire system. Such problem is not yet fully solved for anisotropic thin layers, but some steps have been made in that direction [3], [4].

Luis D. Angulo, M. R. Cabello, and Salvador G. Garcia are with the University of Granada, Granada 18071, Spain. (e-mail: 1mdiazangulo@ugr.es)

David Escot Bocanegra, Patricia Gómez de Francisco, Borja Plaza Gallardo and David Poyatos Martinez are with the National Institute of Aerospace Technology (INTA), in Spain.

The work described in this paper and the research leading to these results have been supported by the Spanish MINECO and EU FEDER under Project TEC2016-79214-C3-1,2,3-R (MINECO, Spain), the EU PRACE project 2018184436 ESECELS, and the Alhambra-LFT contract with AIRBUS (Spain).
The theoretical modeling of thin panels has been addressed by many authors in the past. At low frequencies or when the conducting fibers in the panel are very loose, a simple impedance model can be deduced [2], [5], [6]. However, to have loose fibers is not the most common situation for CFCs. On the contrary, fibers are typically packed very close and the main approach consists on developing an effective permittivity model from the conducting characteristics of fiber and the matrix material [7], [8]. These implicitly assume the existence of a proximity effect between currents induced in the fibers, which dominates the SE at high frequencies. Although valid, these approaches require the use of numerical tools which make very difficult to track the obtained SE to the different design parameters in the CFC, such as the radius and fiber conductivity. Additionally, in these works, predictions are validated with numerical simulations producing very good matches, but always lacking an experimental confirmation.

From an experimental approach, we can find many works measuring CFC panels with a few layers, typically less than four, and SE below $100 \mathrm{~dB}$ [9], [10]. This is typically accomplished using the ASTM method [11] or some variant [12]. The measurement of higher SE has not been reported despite the fact that CFCs of 10 to 30 layers, with arbitrary orientations, are ubiquitously used in vehicles, and can arguably present much higher shielding. The reason for this can be attributed to the lack of a dynamic range in the instruments employed which is typically about $100 \mathrm{~dB}$. These works also lack a connection between the microscopic level and the measured SE. Moreover, the interpretation of experimental results is confined to the fitting of an effective conductivity, which clearly lacks the capability of explaining some of the observed phenomena.

In this context, the aim of this work is to propose and validate a model which allows to connect high frequency (HF) properties with their low frequency (LF) ones, the latter much easier to measure and having a much larger dynamic range. With the help of these models, we devise a new method which can accurately predict and measure much higher SE of CFCs, going beyond the typical frequency ranges, than the ones reported until now.

\section{Modeling METHOD}

A CFC is usually composed of several layers of stacked fibers with different orientations, typically forming angles of 0,45 , or 90 degrees among each other (Fig. 1). These fibers can be weaved or laid straight, the latter being the subject of 


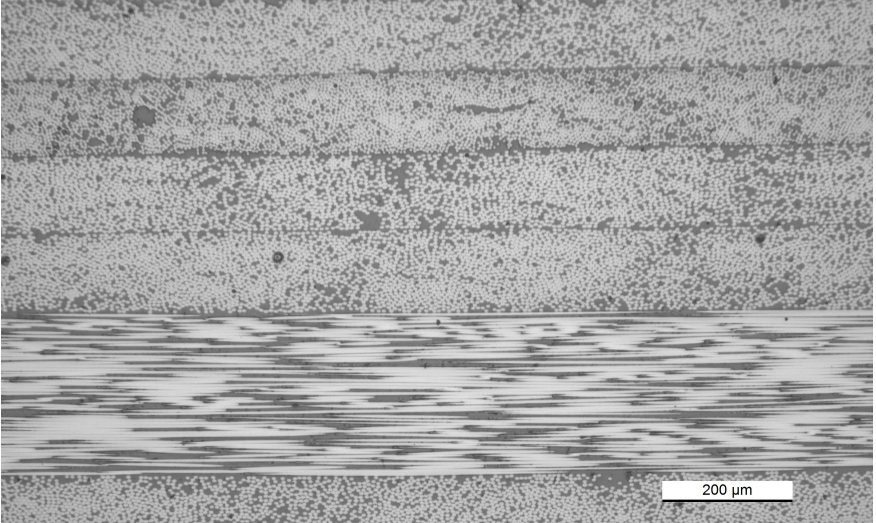

Fig. 1. Microscopic image of a CFC panel cut. Electromagnetic waves are assumed to illuminate the panel coming from top to bottom of the image, or vice-versa. Layers forming 45 or 90 degrees are seen as ellipsoids or lines, respectively.

this work. A non-conductive matrix resin is usually used to cure the panels, gluing the fibers together.

When a harmonic plane-wave, having an incident electric field amplitude $\left(E^{i}\right)$ illuminates perpendicularly a flat panel (Fig. 2), part of it can be reflected $\left(E^{r}\right)$, transmitted $\left(E^{t}\right)$, or absorbed. The SE can be defined as the inverse of the transmission coefficient,

$$
\mathrm{SE}=\frac{1}{|T|}=\frac{\left|E^{i}\right|}{\left|E^{t}\right|}
$$

In order to derive an expression from its geometrical and physical parameters, thin panels can be conceptualized as twoport networks. The fields on one side $\left(E_{1}, H_{1}\right)$ and the other $\left(E_{2}, H_{2}\right)$ are related by

$$
\left[\begin{array}{l}
E_{2} \\
H_{2}
\end{array}\right]=[\Phi]\left[\begin{array}{l}
E_{1} \\
H_{1}
\end{array}\right]=\prod_{i=1}^{N_{l}}\left[\Phi_{i}\right]\left[\begin{array}{l}
E_{1} \\
H_{1}
\end{array}\right]
$$

with $\Phi$ and $\Phi_{i}$ being the transmission matrices, or ABCD matrices, of the whole thin panel and of each of the stacked layers composing it, respectively. For panels located in free-space, characterized by an intrinsic impedance $\eta_{0}$, the transmission coefficient $(T)$ can be obtained as [13],

$$
T=\frac{2 \eta_{0}}{\Phi_{11} \eta_{0}+\Phi_{12}+\Phi_{21} \eta_{0}^{2}+\Phi_{22} \eta_{0}}
$$

Note that Eq. (1) is a scalar, which is sufficient for isotropic media. However, as CFCs are intrinsically anisotropic media, we would need to account for the different polarization modes that illuminate the fiber in a transversal (TE) or parallel (TM) direction. For this kind of anisotropic media, we can define an average SE combining the transmission coefficients as

$$
\mathrm{SE}_{\mathrm{av}}=\frac{2}{\left|T_{\mathrm{TM}}\right|+\left|T_{\mathrm{TE}}\right|}
$$

Please note that (4) does not consider inter-mode conversions which would dominate if two consecutive layers of conductive fibers are not perpendicularly oriented. However, for the sake of simplicity, we do not consider these cases in the remainder of this work.

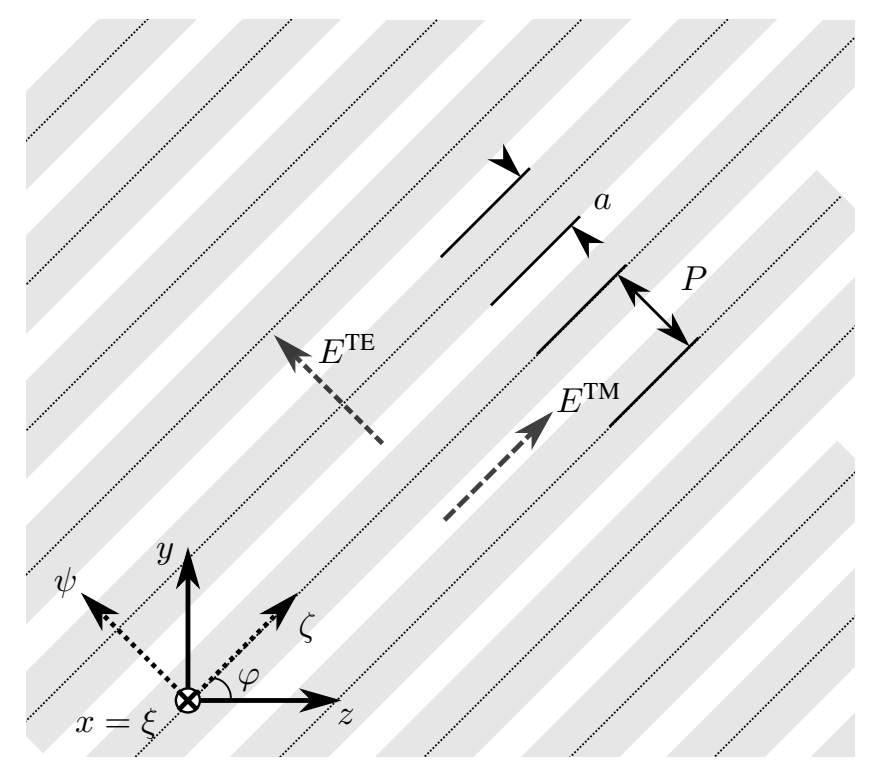

Fig. 2. Geometrical characteristics of the carbon fiber seen from the front: diameter $a$, and period $P$. The representation with local $(\xi, \psi, \zeta)$ and global $(x, y, z)$ coordinate systems

\section{A. Single layer of $C F C$}

As a first approximation, let us assume a CFC composed of a single layer of homogeneously distributed fibers with diameter $a$, infinitely large, and periodically repeated in the plane after a distance $P$. The material which constitutes them is modeled with a conductivity $\sigma_{f}$ which causes them to have an effective permittivity of

$$
\varepsilon_{f}(\omega)=\varepsilon_{0}-j \frac{\sigma_{f}}{\omega}
$$

Fibers are embedded in a dielectric matrix of resin with permittivity $\varepsilon_{m}$ with a total thickness $d$ after curation. Therefore, fibers occupy a relative volume space of

$$
g=\frac{\pi a^{2}}{4 P d}
$$

This mixture of materials can be homogenized using an effective media approach [7] which simplifies their electromagnetic properties to

$$
\begin{aligned}
\varepsilon_{\mathrm{TE}} & =\varepsilon_{m}(1-g)^{-1} \\
\varepsilon_{\mathrm{TM}} & =\varepsilon_{f} g \\
\mu_{\mathrm{TE}} & =\mu_{\mathrm{TM}}=\mu_{0}
\end{aligned}
$$

when $\varepsilon_{f} \gg \varepsilon_{m}$. This simplification is justified when we note that even at frequencies as high as $\sim 100 \mathrm{GHz}$, the conductive term $(\sigma / \omega)$ will dominate over the permittivity in (5) even for conductivities as low as $\sim 6 \Omega / \mathrm{m}$. In [2] we made an study on several models available and found that for most cases this homogenization is sufficient.

From these assumptions, we can now deduce how TE and TM modes are transmitted using the concept of transmission matrix introduced in (2). From [14],

$$
[\Phi]=\left[\begin{array}{cc}
\cosh (\gamma d) & \eta \sinh (\gamma d) \\
\eta^{-1} \sinh (\gamma d) & \cosh (\gamma d)
\end{array}\right]
$$


with $\gamma=j \omega \sqrt{\mu \varepsilon}$ and $\eta=\sqrt{\mu / \varepsilon}$ being the material's complex propagation constant and intrinsic impedance, respectively.

Being anisotropic, the CFC supports two propagation modes, TE and TM. For TE modes the term $\gamma_{\mathrm{TE}} d=$ $j k_{0} d \sqrt{\varepsilon_{m} \varepsilon_{0}^{-1}}$ is negligible for the typical thicknesses of CFC panels at frequencies below several $\mathrm{GHz}$ and a permittivity of a few $\varepsilon_{0}$. In consequence, (8) reduces to the identity matrix and from (3) we obtain that $T_{\mathrm{TE}}=1$.

With the TM mode the situation is very different as $\gamma_{\mathrm{TM}}$ is dominated by the fiber conductivity. This allows $\gamma_{\mathrm{TM}} d$ to be also expressed as a function of the skin depth $\delta=$ $\left(\pi f \mu \sigma_{e}\right)^{-1 / 2}$ with $\sigma_{e}=g \sigma_{f}$ being the effective conductivity, as

$$
\gamma_{\mathrm{TM}} d=(1+j) \frac{d}{\delta}
$$

and the intrinsic impedance as

$$
\eta_{\mathrm{TM}}=\frac{2}{1+j} \frac{1}{\sigma_{e} \delta}
$$

Substituting (8) in (3)

$$
\left|T_{\mathrm{TM}}\right|=\left|\cosh \left(\gamma_{\mathrm{TM}} d\right)+\frac{1}{2} \frac{\eta_{\mathrm{TM}}^{2}+\eta_{0}^{2}}{\eta \eta_{0}} \sinh \left(\gamma_{\mathrm{TM}} d\right)\right|^{-1}
$$

or

$$
\left|T_{\mathrm{TM}}\right|=\left|\frac{\left(\eta_{\mathrm{TM}}+\eta_{0}\right)^{2}}{4 \eta_{\mathrm{TM}} \eta_{0}} e^{\gamma_{\mathrm{TM}} d}-\frac{\left(\eta_{\mathrm{TM}}-\eta_{0}\right)^{2}}{4 \eta_{\mathrm{TM}} \eta_{0}} e^{-\gamma_{\mathrm{TM}} d}\right|^{-1}
$$

Let us now explore Eq. 12 in two frequency regimes. In the LF regime, we can perform a first order Taylor expansion as $e^{ \pm \gamma_{\mathrm{TM}} d \simeq} \simeq 1 \pm \gamma_{\mathrm{TM}} d$. Using (9), (10) on (12); and considering that for conductive panels $|\eta| \ll\left|\eta_{0}\right|$ below frequencies up to hundreds of $\mathrm{GHz}$, we obtain

$$
\left|T_{\mathrm{TM}, \mathrm{LF}}\right|=\left|\frac{\eta_{0} \sigma_{e} d}{2}\right|^{-1}=\left|\frac{1}{2} \frac{\eta_{0}}{R_{\square}}\right|^{-1}
$$

with

$$
R_{\square}=\frac{1}{\sigma_{e} d}
$$

being the DC square resistance of the layer. Note also, that eq. (13) does not depend on the frequency. In the HF regime, the approach $e^{-\gamma_{\mathrm{TM}} d} \simeq 0$ can be made, and (12) takes the form

$$
\left|T_{\mathrm{TM}, \mathrm{HF}}\right|=\left|\frac{\sqrt{2}}{8} \eta_{0} \sigma_{e} \delta e^{\gamma_{\mathrm{TM}} d}\right|^{-1}=\left|\frac{4}{\sqrt{2}} \frac{\eta_{0}}{R_{\square}} \frac{\delta}{d} e^{d / \delta}\right|^{-1}
$$

which is dominated by the exponential term $\gamma_{\mathrm{TM}} d \propto \omega^{1 / 2}$.

To identify the regime in which one or other approach is more pertinent, we can define $w_{c}$ as the corner frequency in which $e^{-\gamma_{\mathrm{TM}} d}=e^{-1}$ (or equivalently $\delta=d \sqrt{2}$ ) resulting in

$$
\omega_{c}=\frac{1}{\mu_{0} \sigma_{e} d^{2}}=\frac{R_{\square}}{\mu_{0} d}
$$

An interesting result which derives from this equation is that the SE of a thicker panel will present a HF exponential behavior at lower frequencies, even if it has the same square resistance as a thinner panel.

Summarizing, from the previous discussion we have reduced the problem of determining the SE of a CFC to knowing only two variables, i.e. the sheet resistance $R_{\square}$ and the thickness $d$. Both, much more easy to obtain experimentally than the exclusively geometric ones.

\section{B. CFCs with an arbitrary number of stacked layers}

Let us consider a simplified case in which we have a stack of CFC layers as the ones described in section II-A forming angles $\varphi$ of 0 or 90 degrees angles with respect to the $z$ axis. We restrict our discussion to just these angles for the simplicity of avoiding the inter-mode terms which would appear in $\Phi$ after applying the Mohr transformations for tensor rotations. For a wave traveling perpendicular to the CFC ( $x$ axis) the fields at both sides can be described with a generalized version of (2),

$$
\left[\begin{array}{c}
E_{y, 2} \\
H_{z, 2} \\
E_{z, 2} \\
-H_{y, 2}
\end{array}\right]=\prod_{i=1}^{N_{l}}\left[\begin{array}{ll}
{\left[\Phi_{\varphi_{i}, i}\right]} & {\left[\Phi_{\varphi_{i}-90^{\circ}, i}\right]}
\end{array}\right]\left[\begin{array}{c}
E_{y, 1} \\
H_{z, 1} \\
E_{z, 1} \\
-H_{y, 1}
\end{array}\right]
$$

where $\left[\Phi_{\varphi_{i}, i}\right]$ corresponds to the $i$-th layer with fiber forming an angle $\varphi_{i}$ with respect to the $z$ axis. For instance, let us assume a wave polarized in the $y$ direction. This implies that $\left[\Phi_{0^{\circ}}\right]$ corresponds to a TM transmission and $\left[\Phi_{90^{\circ}}\right]$ corresponds to a TE transmission.

\section{EXPERIMENTAL SETUPS}

\section{A. ASTM D4935 standard test}

The ASTM Standard D4935 [11] allows the measurement of the SE of a planar material under the action of TEM mode electromagnetic fields in a coaxial line. The use of this mode only allows to measure the SE averaged for all directions, i.e. the measurement of pure TE or TM modes is impossible with this method. The standard ensures that the method is valid over a frequency range of $30 \mathrm{MHz}$ to $1.5 \mathrm{GHz}$ for a normally incident wave. It also provides the dimensions for the specimen holder which is indeed an enlarged, coaxial transmission line with special taper sections and notched matching grooves to maintain a characteristic impedance of $50 \Omega$ throughout the entire length of the holder. Instead of fabricating an exact copy of this flanged-coaxial sample holder (FCSH), we have developed an alternative setup based on commercial connectors and which has been used for this work. This is based on two $31 / 8$ " EIA to $\mathrm{N}$ female adapters from SPINNER with modified inner connectors (Fig. 3). The diameters of the outer and inner conductors of this adapters are $76.9 \mathrm{~mm}$ and $33.4 \mathrm{~mm}$ respectively, what yields a cut-off frequency of $1.73 \mathrm{GHz}$ before overmoding, in agreement with the bounds of the standard.

The signal generator and the receiver described in the standard were substituted for a vector network analyzer (VNA) whose ports 1 and 2 are directly connected through coaxial cables to each of the $\mathrm{N}$ connectors of the adapters. The SE of the material under test (MUT) against a flat TEM wave with normal incidence is then obtained from the measurement of the insertion loss (IL) in the FCSH formed by the two adapters connected to each other. Then, the experimental SE is evaluated as

$$
\mathrm{SE}=20 \log _{10}\left|\frac{S_{21, L}}{S_{21, R}}\right|
$$

where $S_{21, L}$ and $S_{21, R}$ are the measured scattering parameters related to the electromagnetic power transferred from the VNA 


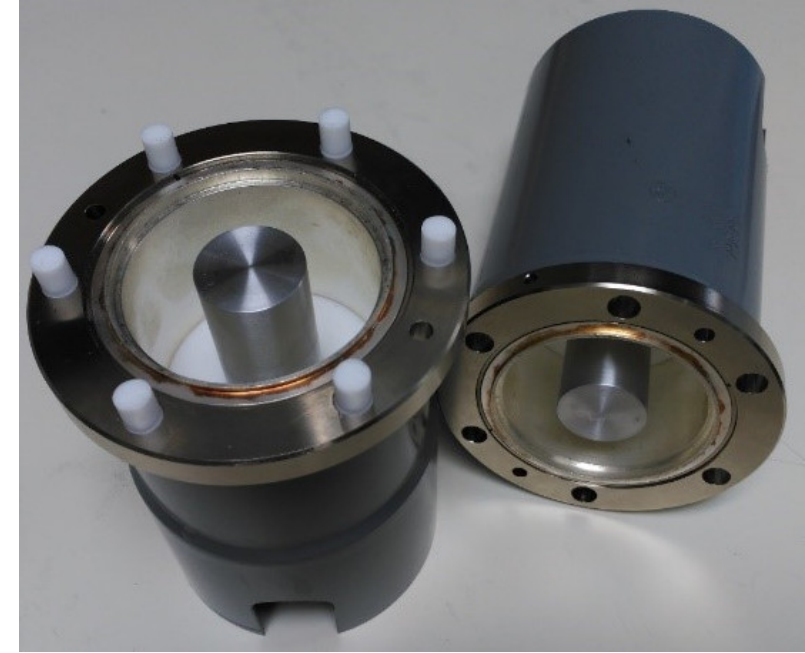

Fig. 3. The two $31 / 8$ " EIA to $\mathrm{N}$ female adapters used in this work as sample holders.

port 1 to the VNA port 2 when the FCSH is filled with the load and reference samples of the specimen, respectively. Thus, two samples are needed for a certain MUT and they must be identical in thickness to get the best repeatability of SE measurements. The standard specifies the dimensions of the reference and load samples for the aforementioned FCSH. It is worth noting that the standard does not mention any need for further manipulation of the samples so they were not modified to improve the electrical contact between them and the coaxial holder, as opposed to [9].

It is important to mention that during the tests, the halves of the cell are connected by means of nylon screws, in order to 1) minimize stray field coupling, 2) reduce the contact resistance with the sample, and 3) improve the capacitive coupling between the two FCSH flanges described in [12]. In order to be sure that the torque given to the screws is always the same, a calibrated dynamometric torque wrench was used.

\section{B. DC resistance test}

In the following setup, we aim to measure the DC square resistance (14) of the samples. This is the resistance between two opposite sides of a square and it is independent of its size. To carry out this test the materials required were: a specimen support, a micro-ohmmeter, and a pneumatic press (Fig. 4). The specimen support consists of two metallic holders separated by two polymer structures fabricated with a $3 \mathrm{D}$ printer. This support makes possible that the current drives only in one direction through the sample (vertically in Fig. 4). To ensure a good contact between the metallic holders and the sample, silver paint was used on the sides of the sample in contact with the holders, previously cleaned with isopropyl alcohol. The painted sample is then placed into the pneumatic press, MEGA PRP20 model [15] with a nominal force of 20 tons in order to warranty enough pressure for an adequate ohmic contact with the fibers and repeatability of measurements. To measure the electrical resistance of the samples, a Keithley 580 micro-ohmmeter [16] was used. This

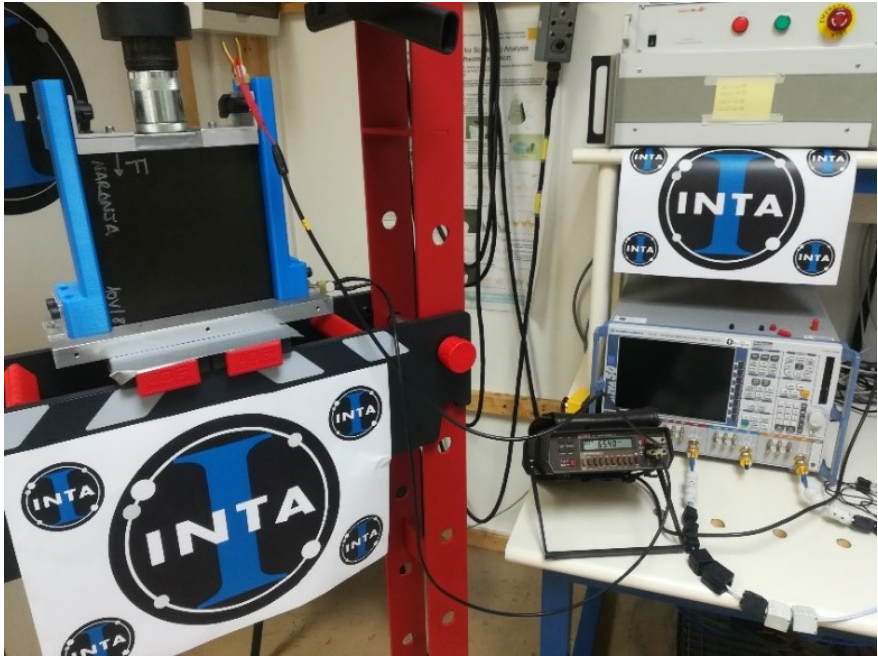

Fig. 4. Test setup for DC resistance measurements. The red parts belong to the hydraulic press used to make pressure on the specimen holded by the blue parts. A micro-ohmmeter was used to measure the square resistance of the sample.

unit is designed for resistance measurement requirements from $10 \mu \Omega$ to $200 \mathrm{k} \Omega$.

The samples used in this setup were $200 \mathrm{~mm}$ long and $200 \mathrm{~mm}$ width, while the thickness depends on the number of CFC layers.

\section{THEORETICAL MODEL AND EXPERIMENTAL CROSS-VALIDATION}

In order to validate the model introduced in section II and the experimental setups described in III we have selected four different samples of CFC. Two samples, Monolayer and Bilayer, have been designed and manufactured with the aim of being as simple as possible in order to simplify their modeling. A second series of two samples, codenamed Red and Blue, is representative of the CFCs used in the MILANO aircraft. The MILANO is a remotely piloted aircraft system developed by INTA and currently in operation. All samples were made with a Cytec MTM 45-1 epoxy matrix and HexTow IM7$12 \mathrm{~K}$ carbon fiber. The particular number of layers and the different orientations used during the stacking sequence of the different samples is summarized in Table I. Every layer is made of unidirectional tows composed of 12000 fibers each, with diameter $a=5.2 \mu \mathrm{m}$ and resistivity $\sigma_{f}^{-1}=1.5 \mathrm{~m} \Omega \cdot \mathrm{cm}$. Having a tow density of three tows per centimeter this adds to $3.6 \times 10^{5}$ fibers $/ \mathrm{m}$. After curation, each layer has a total physical thickness $d_{p}=128 \mu \mathrm{m}$. However, we must distinguish between the physical thickness and the effective thickness, $d$. We define $d$ as the distance of fiber material traversed by the wave in which the fiber is aligned with the electric field (TM mode). Using Eq. (14) we obtain a predicted value of $R_{\square, l}=196 \mathrm{~m} \Omega$ for each of these layers.

The Monolayer sample is composed of a single layer as the one described previously. In this case, the $R_{\square}$ value could not be measured with the DC resistance test because being so thin and weak, the layer could not be warranted an attachment to the holder without being damaged. To compute its SE, we 
TABLE I

MEASURED SAMPLES

\begin{tabular}{l|r|r} 
Name & Stacking Sequence & Layers \\
\hline Monolayer & 0 & 1 \\
Bilayer & $0 / 90$ & 2 \\
Red & $45 /-45 / 0 /-45 / 45 / 90 / 90 / 45 /-45 / 0 /-45 / 45$ & 12 \\
Blue & $45 /-45 /-45 / 0 / 45 / 90 / 45 / 0 /-45 /-45 / 0 / 45 / 90 / 45 / 0 / 45 /-45 / 45$ & 18 \\
\hline
\end{tabular}

TABLE II

MEASURED AND PREDICTED VALUES

\begin{tabular}{l||r||r|r||r|r|r} 
Name & $d[\mu \mathrm{m}]$ & Pred. $R_{\square}[\mathrm{m} \Omega]$ & Meas. $R_{\square}[\mathrm{m} \Omega]$ & Pred. SE $\mathrm{LF}_{\mathrm{LF}}[\mathrm{dB}]$ & Meas. SE $\mathrm{LF}_{\mathrm{LF}}[\mathrm{dB}]$ & Pred. $f_{c}[\mathrm{GHz}]$ \\
\hline Monolayer & $128 / 0$ & $196 / \infty$ & - & 6.0 & $5.7 \pm 0.3$ & 1.220 \\
Bilayer & 128 & 196 & - & 59.6 & $57.1 \pm 0.5$ & 1.220 \\
Red & 768 & 98 & $99.6 \pm 6.8$ & 75.2 & - & 0.033 \\
Blue & 1152 & 49 & $46.4 \pm 0.4$ & 78.7 & - & 0.015 \\
\hline
\end{tabular}

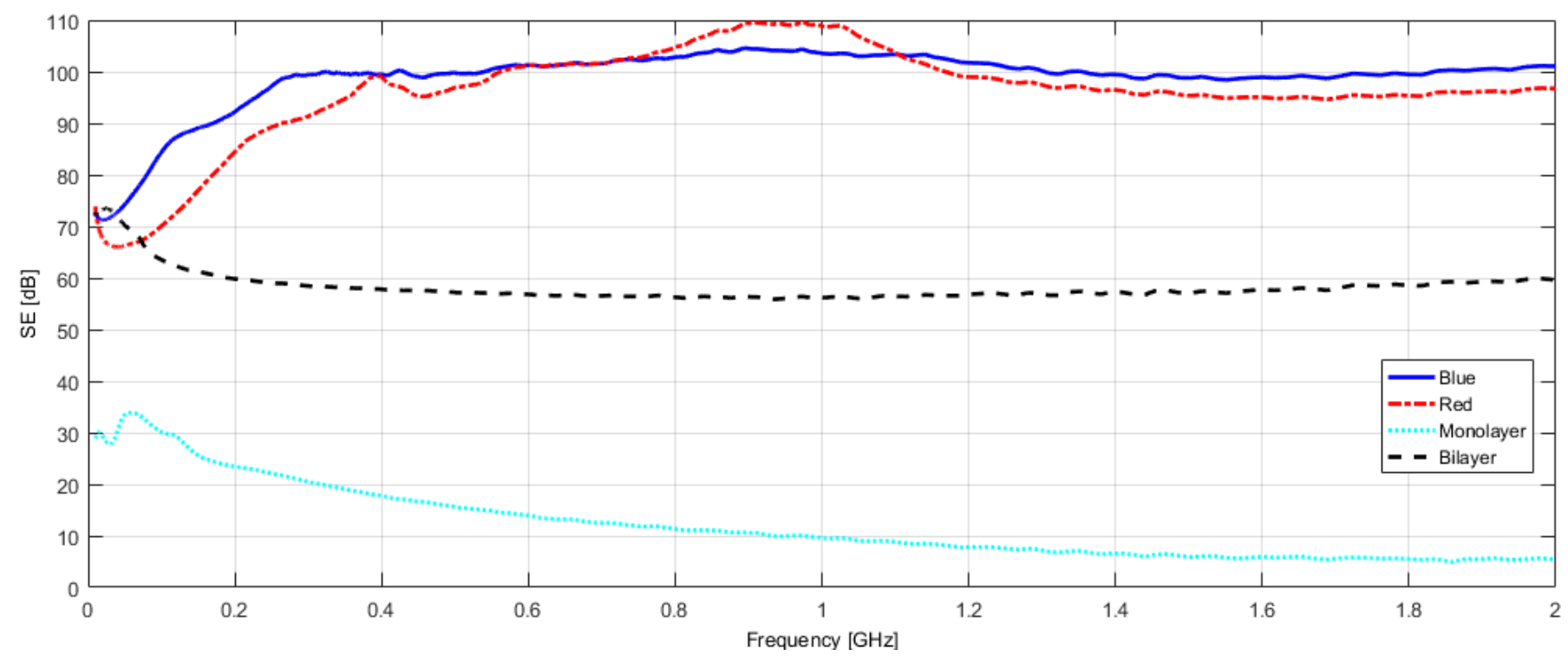

Fig. 5. SE experimental results obtained with the application of the modified version of ASTM D4935 standard described in section III-A.

realize that $T_{\mathrm{TE}}=1$ is much larger than $T_{\mathrm{TM}}$. Therefore Eq. (4) gives a value of $\mathrm{SE}_{\mathrm{av}}=2=6 \mathrm{~dB}$ (Table II). In Fig. 5 we observe the experimental results for the SE of this sample and we can realize that after $\sim 1.5 \mathrm{GHz}$ they tend to the predicted valued of $6 \mathrm{~dB}$. The deviation from the constant value in the range from $0.1 \mathrm{GHz}$ to $1.5 \mathrm{GHz}$ can be attributed to the anisotropic nature of the sample. This effect was corroborated by a simulation using the HFSS software [17], a commercial Finite Element Method numerical solver. Below $0.1 \mathrm{GHz}$ the deviation corresponds to the lost of capacitive coupling between the samples and the FCSH which makes impossible any transmission [11], possibly mixed with the capacitance stray effects reported in [12].

The Bilayer sample combines two layers perpendicularly laid. Using (16) we find that non-constant effects should be observed beyond $1.220 \mathrm{GHz}$, as the data in Fig. 5 starts to faintly suggest. In this case we find that $T_{\mathrm{TE}}=T_{\mathrm{TM}}$ after calculating the transmission matrix in (17). In consequence at $\mathrm{LF}$, the SE is simply

$$
\mathrm{SE}_{\mathrm{LF}}=\frac{2 R_{\square}}{\eta_{0}}=59.6 \mathrm{~dB}
$$

which greatly coincides with the measured value of $57.1 \pm$ $0.5 \mathrm{~dB}$. We can also observe that, again, and mainly below $0.1 \mathrm{GHz}$, the ASTM standard fails to provide reliable measurements and tends to overestimate the SE, as mentioned in its introduction [11].

The Red and Blue samples are made of 12 and 18 layers, stacked perpendicularly with their neighbors in most cases. The cases in which the transitions are not perpendicular would lead us to expect some inter-modal conversions which may modify the assumptions made in section II but which we will ignore as they fall beyond what we can measure in the scope of this work. The square resistance of these samples was measured using the setup described in section III-B finding values that corroborate the square resistance predictions made from pure geometrical considerations (Table II). The same assumptions as with the Bilayer case were made to predict $\mathrm{SE}_{\mathrm{LF}}$ values of $75.2 \mathrm{~dB}$ and $78.7 \mathrm{~dB}$ for the Red and Blue samples, respectively. However, when we compute their corner frequencies using (16), we predict that this LF regime ends at $33 \mathrm{MHz}$ and $15 \mathrm{MHz}$, respectively. This means that above those frequencies we must observe a $\mathrm{SE}$ which grows exponentially with $\omega^{1 / 2}$. This is what we observe in the range 
up to $0.4 \mathrm{GHz}$ just before the values escape the dynamic range of the VNA. As in the previous cases, the ASTM standard does not provided reliable results below $0.1 \mathrm{GHz}$. This, together with the very low expected value for $f_{c}$ makes impossible to provide a measurement for $\mathrm{SE}_{\mathrm{LF}}$ and we are left only with the theoretical predictions which set an inferior limit for the samples.

\section{CONCLUSiOns}

In this work we have provided a model able to predict the SE of some CFC panels representative of the ones used in real aircrafts (Sec. II). This modeling permitted us to obtain simple formulas in which the only parameters needed to make predictions are the sheet square resistance and the effective panel thickness; both easy to obtain with inexpensive equipment. These tools let us to predict a minimum, or worst case, SE. This minimum value is always at LF and always increases with the frequency. In consequence, the measurement of minimum SE requirements can be deduced simply with a micro-ohmmeter using the experimental set-up described in section III-B.

\section{REFERENCES}

[1] G. G. Gutierrez, D. Mateos, M. R. Cabello, E. Pascual-Gil, L. D. Angulo, and S. G. Garcia, "On the design of aircraft electrical structure networks," IEEE Transactions on Electromagnetic Compatibility, vol. 58, no. 2, pp. 401 - 408, March 2016.

[2] L. D. Angulo, M. R. Cabello, J. Alvarez, A. R. Bretones, and S. G. Garcia, "From microscopic to macroscopic description of composite thin panels: A roadmap for their simulation in time domain," IEEE Transactions on Microwave Theory and Techniques, vol. 66, no. 2, 2018.

[3] M. R. Cabello, L. D. Angulo, J. Alvarez, A. R. Bretones, and S. G. Garcia, "Subgridding boundary conditions to model arbitrarily dispersive thin planar materials," IEEE Transactions on Antennas and Propagation, vol. 66, no. 11, pp. 6429-6434, Nov 2018.

[4] M. R. Cabello, L. D. Angulo, J. Alvarez, A. R. Bretones, and S. G. Garcia, "A new conformal fdtd for lossy thin panels," IEEE Transactions on Antennas and Propagation, pp. 1-1, 2019.

[5] M. S. Sarto, S. Greco, and A. Tamburrano, "Shielding effectiveness of protective metallic wire meshes: EM modeling and validation," IEEE Transactions on Electromagnetic Compatibility, vol. 56, no. 3, pp. 615621, June 2014

[6] V. Volski and G. A. Vandenbosch, "Full-wave electromagnetic modelling of fabrics and composites," Composites Science and Technology, vol. 69, no. 2, pp. 161 - 168, 2009. [Online]. Available: http://www.sciencedirect.com/science/article/pii/S0266353808003825

[7] C. Holloway, M. Sarto, and M. Johansson, "Analyzing carbon-fiber composite materials with equivalent-layer models," Electromagnetic Compatibility, IEEE Transactions on, vol. 47, no. 4, pp. 833-844, Nov 2005.

[8] V. Préault, R. Corcolle, L. Daniel, and L. Pichon, "Effective permittivity of shielding composite materials for microwave frequencies," IEEE Transactions on Electromagnetic compatibility, vol. 55, no. 6, pp. 11781186, 2013.

[9] S. Greco, A. Tamburrano, A. D'Aloia, R. Mufatti, and M. S. Sarto, "Shielding effectiveness properties of carbon-fiber reinforced composite for HIRF applications," in International Symposium on Electromagnetic Compatibility - EMC EUROPE, Sept 2012, pp. 1-6.

[10] D. Munalli, G. Dimitrakis, D. Chronopoulos, S. Greedy, and A. Long, "Electromagnetic shielding effectiveness of carbon fibre reinforced composites," Composites Part B: Engineering, vol. 173, p. 106906, 2019. [Online]. Available: http://www.sciencedirect.com/science/article/ pii/S1359836818343762

[11] Standard Test Method for Measuring the Electromagnetic Shielding Effectiveness of Planar Materials, ASTM International Std. D4935-18, 2018.
[12] A. Tamburrano, D. Desideri, A. Maschio, and M. S. Sarto, "Coaxial waveguide methods for shielding effectiveness measurement of planar materials up to $18 \mathrm{GHz}$," IEEE Transactions on Electromagnetic Compatibility, vol. 56, no. 6, pp. 1386-1395, 2014.

[13] D. Frickey, "Conversions between S, Z, Y, H, ABCD, and T parameters which are valid for complex source and load impedances," IEEE Transactions on Microwave Theory and Techniques, vol. 42, no. 2, pp. 205-211, 1994

[14] S. J. Orfanidis, Electromagnetic waves and antennas, 2002.

[15] [Online]. Available: https://www.mega.es

[16] [Online]. Available: http://www.testequipmenthq.com/datasheets/ KEITHLEY-580-Datasheet.pdf

[17] [Online]. Available: https://www.ansys.com/products/electronics/ ansys-hfss 